\title{
Long-term experience with implantable treprostinil pumps in pulmonary arterial hypertension
}

\author{
Dytrych $\mathrm{V}^{1}$, Kuchar $\mathrm{J}^{2}$, Ambroz $\mathrm{D}^{1}$, Kunstyr $\mathrm{J}^{3}$, Grus $\mathrm{T}^{4}$, Lindner $\mathrm{J}^{4}$, Linhart $\mathrm{A}^{1}$, Jansa $\mathrm{P}^{1}$ \\ Clinical Department of Cardiology and Angiology, 1st Faculty of Medicine, 2nd Medical Department, \\ Charles University, Praha, Czech Republic. pavel.jansa@vfn.cz
}

\begin{abstract}
OBJECTIVES: To compare clinical parameters and quality of life in patients with pulmonary arterial hypertension (PAH) at the time of diagnosis, at the time of LenusPro pump implantation and during intravenous treptostinil treatment.

METHODS: Seven patients with severe PAH treated with intravenous treptostinil via implantable LenusPro pumps were evaluated, including NYHA classification, six-minute walking test, BNP and quality of life assessment using the EQ-5D-5L questionnaire before and after pump implantation.

RESULTS: No significant changes were observed in NYHA class and six-minute walking distance test. There was however a significant improvement in the quality of life and a decrease in BNP levels. The mean EQ-5D$5 \mathrm{~L}$ index assessed during subcutaneous treptostinil treatment was significantly worse when compared to that assessed during its intravenous application $(0.39 \pm 0.24$ vs $0.78 \pm 0.28, p<0.05)$; the same is true about the pain/discomfort dimension. Complications occurred, namely one nonfatal pneumothorax, one nonfatal hemothorax, and one event of nonfatal treptostinil intoxication after refilling.

CONCLUSIONS: In patients who do not tolerate subcutaneous treptostinil treatment, the use of the LenusPro implantable pump results in a significant improvement in quality of life with an acceptable safety profile (Tab. 2, Fig. 2, Ref. 19). Text in PDF www.elis.sk. KEY WORDS: pulmonary arterial hypertension, treptostinil, implantable pump, LenusPro, quality of life.
\end{abstract}

\section{Introduction}

Pulmonary arterial hypertension (PAH) is a chronic progressive disease leading to right-sided heart failure and ultimately to death if untreated. PAH can only be diagnosed by right heart catheterization (RHC) and is defined as mean pulmonary arterial pressure (PAMP) $\geq 25 \mathrm{mmHg}$, pulmonary capillary wedge pressure $(\mathrm{PCW}) \leq 15 \mathrm{mmHg}$, and pulmonary vascular resistance $(\mathrm{PVR})>$ 3 Wood Units (1).

Parenteral prostanoids are indicated for high-risk patients with pulmonary arterial hypertension (PAH). This treatment improves hemodynamic parameters, functional class and six-minute walking test (6MWT) as well as survival (1-2). Intravenous treatment

12nd Department of Medicine - Department of Cardiovascular Medicine, First Faculty of Medicine, Charles University and General University Hospital in Prague, Czech Republic, ${ }^{2}$ Department of Cardiology, Tabor Hospital, Inc., Czech Republic, ${ }^{3}$ Department of Anesthesiology, Resuscitation and Intensive Medicine, First Faculty of Medicine, Charles University and General University Hospital in Prague, Czech Republic, and ${ }^{4} 2$ nd Department of Surgery - Department of Cardiovascular Surgery, First Faculty of Medicine, Charles University and General University Hospital in Prague, Czech Republic

Address for correspondence: P. Jansa, Clinical Department of Cardiology and Angiology, 1st Faculty of Medicine, 2nd Medical Department, Charles University, U Nemocnice 2, CZ-128 08 Praha 2, Czech Repubic. Phone: +420.2 .24962630 , Fax. +420.2 .24912154$

Acknowledgements: Supported by the by the research project Progres Q38/LF1. with the first-generation prostacyclin analog, epoprostenol, administered by an external intravenous pump is highly effective but may be accompanied by severe complications, especially catheter-related infections. In addition, epoprostenol has a very short half-life of 3-5 minutes and the discontinuation of treatment is associated with a rebound phenomenon bearing the risk of a potentially fatal hemodynamic deterioration. Many studies have also shown a high efficacy of treatment with another prostacyclin analog, treprostinil (3-7). The advantage of treprostinil is in its significantly longer half-life, allowing subcutaneous administration by an external pump. However, in 5-10\% of patients, this treatment has to be discontinued due to a strong local reaction associated with significant pain at the application site. The intravenous administration of treprostinil via an implantable Lenus Pro pump may be a suitable solution for such patients (8-10). The pump is implanted subcutaneously in the right upper abdominal quadrant, and connected to a tunneled catheter leading into the superior vena cava. The pump contains a reservoir attached to a septum with a silicone refill access. The usual reservoir capacity is $40 \mathrm{ml}$. A constant flow rate from $1.3 \mathrm{ml}$ to $2 \mathrm{ml} / 24 \mathrm{~h}$ is generated by a gas-powered microinfusion system and regulated by a manufactured chip capillary. No battery pack is therefore needed for generating the flow (8). The pumps with a reservoir volume of $40 \mathrm{ml}$ are refilled every 28 days on average. The dosage can be adjusted by using various concentrations of treprostinil loaded into the pump reservoir. So far, several papers have shown that the pump implantation is relatively safe with a low risk of periprocedural 
complications (11-14). The long-term treatment has been also shown to be safe while complications such as local infections at the site of pump implantation, catheter obstruction associated with the risk of discontinuation of treprostinil therapy, and increased pump output associated with the risk of an increased amount of applied treprostinil due to a pump failure are relatively rare. However, the administration of treprostinil outside the pump is associated with the risk of acute overdose, which may lead to hemodynamic collapse. Therefore, the pump refilling should only be performed in specialized centers (15). Kurzyna et al (16) described a significant subjective improvement in vitality and physical aspects of the health-related quality of life resulting from the use of the LenusPro implantable pump. In this article, we present our experience with LenusPro implantable pumps in our pulmonary hypertension center and describe clinical parameters and quality of life at the time of diagnosis, during subcutaneous treprostinil treatment and during intravenous treprostinil treatment after the pump implantation.

\section{Methods}

This is a retrospective study including 7 patients $(5$ females and 2 males) with severe PAH ( 4 cases of idiopathic PAH, 2 con-
Tab. 1. Baseline characteristics at the time of diagnosis.

\begin{tabular}{lc}
\hline Females N (\%) & $5(71)$ \\
\hline Age (years) & $39 \pm 14$ \\
PAMP (mmHg) & $65.3 \pm 12$ \\
PCWP (mmHg) & $10.6 \pm 3.2$ \\
PVR (WU) & $12.6 \pm 5.2$ \\
6MWD (m) & $441 \pm 78$ \\
NYHA FC N ((I/II/III/IV) & $0 / 3 / 4 / 0$ \\
\hline
\end{tabular}

mean $\pm \mathrm{SD}, \mathrm{PAMP}$ - mean pulmonary artery pressure, $\mathrm{PCWP}$ - pulmonary capillary wedge pressure, PVR - pulmonary vascular resistance, WU - Wood units, 6MWD - six-minute walking distance, NYHA FC

genital heart disease-associated PAH, 1 drug-induced PAH; all patients in NYHA functional class (FC) III, PAMP $65 \pm 12 \mathrm{mmHg}$, PVR $12.6 \pm 5.2 \mathrm{mmHg}$ at the baseline) treated with intravenous treptostinil via implantable LenusPro pumps (Tab. 1). The median time of treatment with subcutaneous treprostinil prior to the pump implantation was 11 months. Clinical evaluation including NYHA classification, six-minute walking test, BNP and assessments of the quality of life using the EQ-5D-5L questionnaire were performed at the time of diagnosis, during subcutaneous treprostinil treatment, and during intravenous treprostinil treatment via implantable LenusPro pump. EQ-5D-5L is a standardized measure of
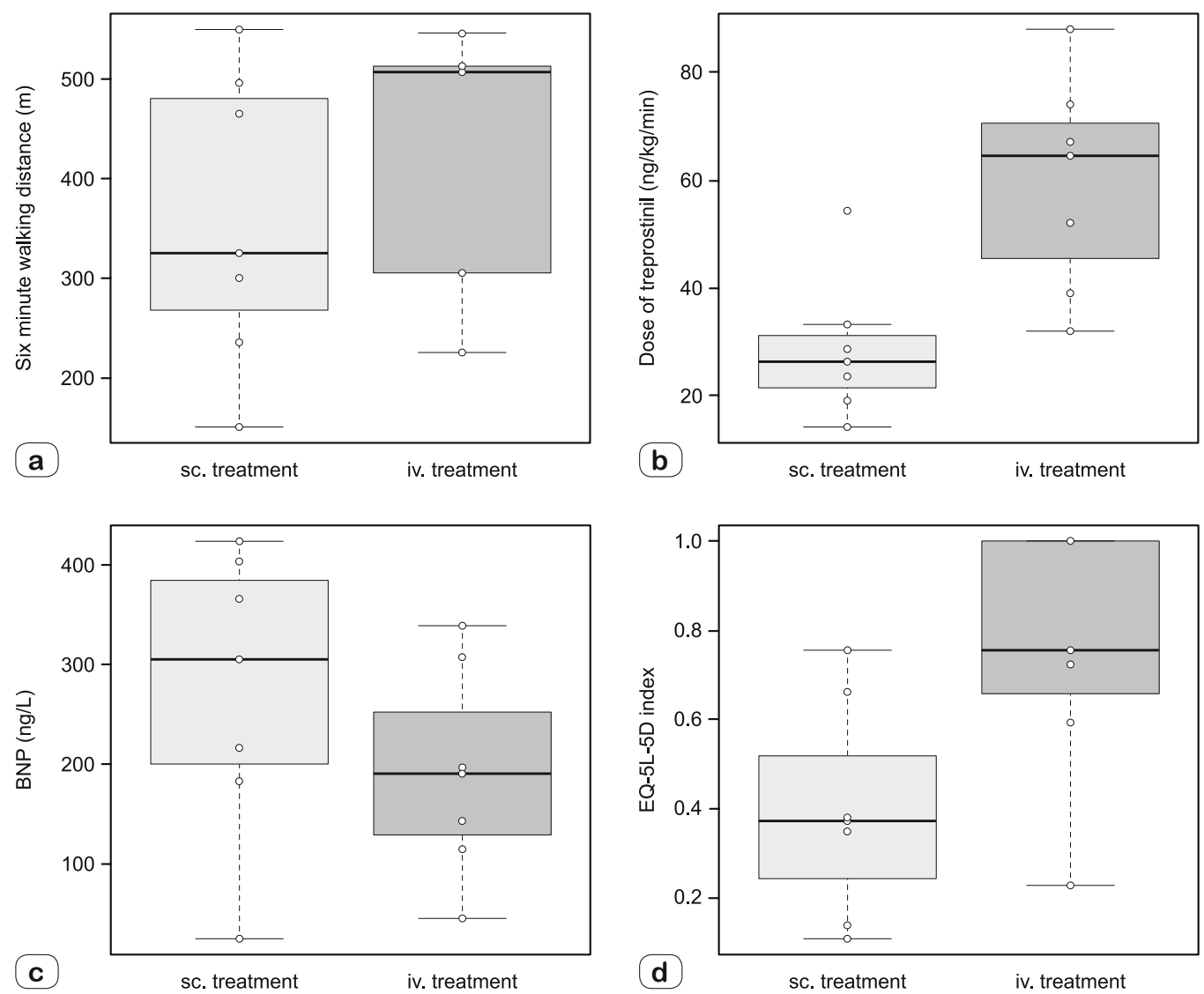

Fig. 1. Changes in six-minute walking distance test, dose of treprostinil, BNP levels and EQ-5D-5L index between sc. treprostinil therapy (before pump system implantation) and i.v. treprostinil therapy (after pump system implantation). No change was observed in six-minute walking distance test (a). The doses of treprostinil were significantly higher after the implantation (b). BNP levels were significantly lower (c) and EQ-5D-5L index was significantly higher (d) after pump system implantation. 


\section{8-361}

health developed by the EuroQol group. It evaluates the following five aspects of health: mobility, self-care, usual activities, pain/ discomfort and anxiety/depression. Each dimension contains five response levels of severity. EQ-5D-5L health states can be summarized using a 5-digit code or be represented by a single calculated number (index value), which reflects how good or bad the health condition is. The higher the index, the better the health state (17).

The statistical analysis was performed using R-project, version 3.2.3. Results are expressed as mean and standard deviation for continuous variables or as number of cases and percentages for categorical variables. T-test was used for comparison of normally distributed variables and Shapiro-Wilk test for normality testing. Values of $p<0.05$ were considered statistically significant.

The study was conducted in accordance with the principles laid down in the 18th World Medical Assembly (Helsinki, 1964), including all subsequent amendments, and in compliance with all laws and regulations of the Czech Republic. The approval of the retrospective data collection was provided by the Ethics Committee of the General University Hospital in Prague.

\section{Results}

The median duration of treatment with implantable pump systems was 29 months.

After the implantation, no changes were observed in NYHA FC (it remained unchanged in all patients) and in the six-minute walking distance test (the results are missing in two patients; mean difference of $+37.8 \mathrm{~m} ; 95 \%$ CI of the difference -107 to $+183 ; \mathrm{p}=$ $0.51)$. However, after the implantation, the doses of treprostinil were significantly higher than before the implantation (mean difference of $+31.0 \mathrm{ng} / \mathrm{kg} / \mathrm{min} ; 95 \% \mathrm{CI}+10.1$ to $+51.9 ; \mathrm{p}=0.01)$ and there was a significant decrease in BNP levels (mean difference of $-84 \mathrm{ng} / \mathrm{L}, 95 \% \mathrm{CI}-10.0$ to -158.3 ; $\mathrm{p}=0.03)$. A significant improvement was also recorded in the quality of life, as assessed by EQ-5D-5L index (mean difference $+0.363,95 \%$ CI 0.004-0.722, $\mathrm{p}=0.048)$ (Tab. 2, Fig. 1).

In comparison with the subcutaneous treatment, an improvement was recorded in all modalities of the EQ-5D-5L questionnaire, including a significant relief from pain/discomfort (Fig. 2). As far as complications are concerned, we recorded one case of nonfatal pneumothorax, one case of hemothorax after pump implantation, and one event of nonfatal treptostinil intoxication after refilling (out of approx. 300 refill procedures).

Tab. 2. Change in NYHA FC, 6MWT, treprostinil dose and BNP after LenusPro Pump implantation.

\begin{tabular}{lccc}
\hline & $\begin{array}{c}\text { Before } \\
\text { implantation }\end{array}$ & $\begin{array}{c}\text { After implantation } \\
\text { (median 29 months } \\
\text { after implantation) }\end{array}$ & $\mathrm{p}$ \\
\hline NYHA FC N (I/II/III/IV) & $0 / 0 / 7 / 0$ & $0 / 0 / 7 / 0$ & - \\
6MWT (m) & $360 \pm 147$ & $419 \pm 144$ & 0.51 \\
Treprostinil dose (ng/kg/min) & $28.5 \pm 13$ & $59.5 \pm 19.7$ & 0.01 \\
BNP (ng/L) & $275 \pm 144$ & $191 \pm 105$ & 0.032 \\
EQ-5D-5L index & $0.394 \pm 0.243$ & $0.757 \pm 0.284$ & 0.048 \\
\hline mean \pm SD, NYHA FC, 6MWD - six-minute walking distance, BNP - brain na- \\
triuretic peptide
\end{tabular}

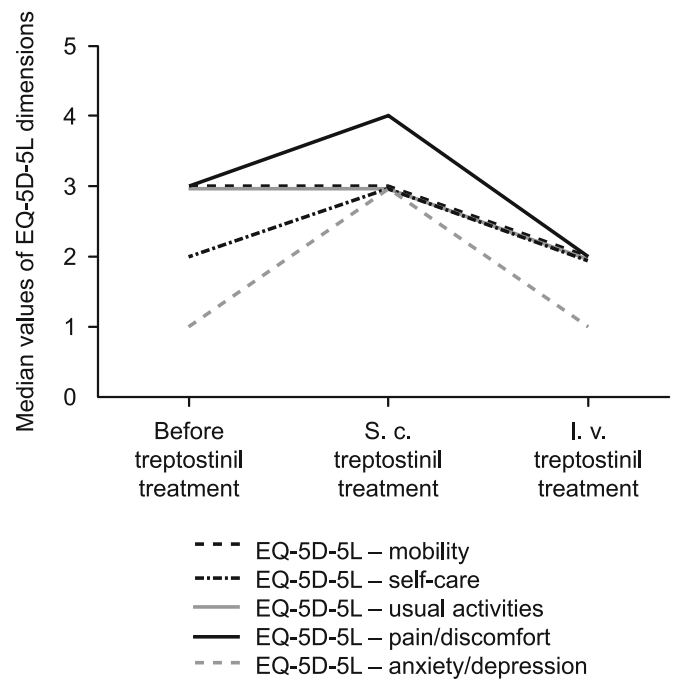

Fig. 2. Medians of individual dimensions of EQ5D5L in treated patients before s.c. treprostinil therapy initiation, during s.c. treprostinil therapy and during i.v. treprostinil therapy (median 29 months after pump system implantation); A smaller number means better quality in individual dimensions.

\section{Discussion}

Parenteral prostanoid therapy is indicated in patients with advanced forms of PAH whose prognosis is generally poor (4). Prior to the development of PAH-specific drug therapies, the median survival rate in the first published registry recording of newly diagnosed patients with idiopathic PAH in 1991 was 2.8 years, with survival rates of $68 \%, 48 \%$, and $34 \%$ at 1,3 , and 5 years, respectively. One prospective randomized trial with epoprostenol and many retrospective studies and registries with epoprostenol and treprostinil have shown a significant improvement in patient survival after prostanoid therapy and there is currently no doubt that prostanoid treatment is effective not only in improving patient symptoms but also in improving survival. In one registry with 860 subcutaneous treprostinil-treated pulmonary arterial hypertension patients, the survival estimates were $87 \%$ at 1 year, $78 \%$ at 2 years, $71 \%$ at 3 years and $68 \%$ at 4 years. Moreover, the discontinuation of treprostinil due to treatment-related adverse events, particularly severe pain at the injection site, was associated with a very poor prognosis, which was significantly worse than in patients who continued with the treatment (18-19).

All patients in our group had advanced functional disability and were in NYHA FC III. In all patients, the pump implantation was preceded by treatment with subcutaneous treprostinil by an external pump, and the indication of pump implantation was a pronounced local injection site reaction associated with severe pain.

The functional class remained the same during both treatments and no significant difference in 6MWT was observed (Tab. 2). The increase in the treptostinil dose and reduction in BNP values were however statistically significant (Tab. 2, Fig. 1).

Our study demonstrated the relative safety of pump implantation under general anesthesia. The most serious complications in 
our group were non-fatal pneumothorax and hemothorax in two patients after pump implantation. Moreover, it should be kept in mind that patients with advanced PAH represent a group at highrisk for general anesthesia. Several papers have shown that the procedure may be associated with fatal complications, especially unmanageable right-sided heart failure (11-14). It is therefore crucial that the procedure is performed in centers experienced in the treatment of pulmonary hypertension and that the general anesthesia is conducted by an experienced anesthetist. Like other papers, our study has shown that intravenous treprostinil treatment via an implantable pump is at least as effective as subcutaneous treprostinil treatment. Many complications described in other works have not occurred in our group of patients, namely catheter dislocation associated with the risk of atrial flutter or atrial fibrillation, catheter obstruction or pump failure, which in some cases led to increased flow and treprostinil overdose (15), as well as catheter-related or local infections. The most serious complication in our cohort (other than the above-mentioned perioperative complications) was one event of off-pump refilling associated with acute intoxication with treprostinil, leading to non-fatal acute myocardial infarction. It is necessary to consider that it was one failure out of approx. 300 refilling procedures, i.e., the failure rate is only approx. $0.3 \%$. Nevertheless, this complication has to be kept in mind as it emphasizes the necessity for the refilling of the pump to be performed by experienced physicians at a pulmonary hypertension center.

As demonstrated by a number of studies, the treatment with subcutaneous treprostinil is effective in improving hemodynamic parameters, 6MWT and functional class (3-4). Yet, paradoxically, it may be at the same time associated with deteriorated quality of life due to the pain associated with its subcutaneous application.

However, it should be emphasized that only patients with severe local reactions and pain were indicated for the intravenous treprostinil treatment via implantable pump. Therefore, it cannot be concluded in general that the treatment with subcutaneous treprostinil deteriorates the quality of life in all patients. Our study has, however, shown that even patients with severe local reactions and pain, in whom the treatment with treprostinil would be probably withdrawn due to this complication could still benefit from this treatment. The absence of local pain facilitated a more effective treatment owing to the possibility of increasing the dose and benefiting from the resulting reduction in BNP levels.

\section{Conclusion}

In patients who do not tolerate subcutaneous treptostinil treatment, the use of the LenusPro implantable pump results in a significant improvement in their quality of life, acceptable safety profile and better control of the disease demonstrated by the decrease in BNP levels.

\section{Learning points}

- Some patients do not tolerate the subcutaneous application of treptostinil in the treatment of pulmonary arterial hypertension.

- The application of an implantable pump allowed such patients to continue with their very effective therapy.
- The quality of life improved in these patients.

- The implantation and refilling of the pump must be performed in specialized centers.

\section{References}

1. Galiè N, Humbert M, Vachiery JLet al. 2015 ESC/ERS guidelines for the diagnosis and treatment of pulmonary hypertension. Eur Heart J 2016; 37: 67-119.

2. Šimková I, Pacák J, Riečanský I et al. Initial experiences with novel therapy for pulmonary hypertension in Slovakia : Klinická štúdia. Bratisl Med J 2006; 107: 239-247.

3. Jansa $\mathbf{P}$, Ambroz D, Maresova $\mathbf{J}$ et al. Pulmonary arterial hypertension - contemporary management strategy. Bratisl Med J 2009, 110: 603-608.

4. Galié N, Channick RN, Frantz RP et al. Risk stratification and medical therapy of pulmonary arterial hypertension. Eur Respir J 2019; 53 (1): 180-189.

5. Barnes H, Yeoh HL, Fothergill T, Burns A et. al. Prostacyclin for pulmonary arterial hypertension. Cochrane. Database Syst Rev 2019; 5: CD012785.

6. Lindegaard Pedersen M, Krüger M, Grimm D et al. The prostacyclin analogue treprostinil in the treatment of pulmonary arterial hypertension. Basic Clin Pharmacol Toxicol 2019; 00: 1-11.

7. Kimmig LM, LiaoC, Bag R. Ambulatory Transition from Parenteral Prostanoid to Inhaled Treprostinil in Patients with Pulmonary Arterial Hypertension. Lung 2020. doi:10.1007/s00408-019-00306-4 .

8. Steringer-Mascherbauer R, Eder V, Ebner $\mathrm{C}$ et al. First experiences with intravenous Treprostinil delivered by an implantable pump (Lenus pro $($ ) with filling intervals of 28 days in patients with pulmonary arterial hypertension (PAH) - a series of five cases Chest 2011; 140: 904A.

9. Ewert R, Halank M, Bruch L, Ghofrani HA. A case series of patients with severe pulmonary hypertension receiving an implantable pump for intravenous prostanoid therapy Am J Respir Crit Care Med 2012; 186: 1196-1198.

10. Ewert R, Richter MJ, Steringer-Mascherbauer R et al. Intravenous treprostinil infusion via a fully implantable pump for pulmonary arterial hypertension. Clin Res Cardiol 2017; 106: 776-783.

11. Waxman AB, McElderry HT, Gomberg-Maitland M et al. Totally Implantable IV Treprostinil Therapy in Pulmonary Hypertension Assessment of the Implantation Procedure. Chest 2017; 152: 1128-1134.

12. Steringer-Mascherbauer R, Eder V, Huber C et al. Intravenous treprostinil delivered by the implantable pump Lenus pro®: a innovative "surgical" approach to management of PAH. J Heart Lung Transplant 2013; 37: S64.

13. Desole $\mathbf{S}$, Velik-Salchner $\mathbf{C}$, Fraedrich $\mathbf{G}$ et al. Subcutaneous implantation of a new intravenous pump system for prostacyclin treatment in patients with pulmonary arterial hypertension. Heart Lung 2012; 41 (6): 599-605.

14. Gomberg-Maitland M, Bourge RC, Shapiro SM et al. Long-term results of the DeIIvery for Pulmonary Arterial Hypertension trial. Pulm Circ 2019; 9 (4): 2045894019878615.

15. Richter MJ, Harutyunova S, Bollmann T et al. Long-term safety and outcome of intravenous treprostinil via implanted pump in pulmonary hypertension. J Heart and Lung Transplant 2018; 37 (10): 1235-1244.

16. Kurzyna M, Rajpold AM, Koteja A et al. An implantable pump Lenus pro $®$ in the treatment of pulmonary arterial hypertension with intravenous treprostinil. BMC Pulmonary Med 2017; 17: 162.

17. Reenen van M. (April 2015). "EQ-5D-5L User Guide". EQ-5D. EuroQol Research Foundation.

18. McLaughlin VV, Shah SJ, Souza R et al. Management of pulmonary arterial hypertension. J Am Coll Cardiol 2015; 65: 1976-1997.

19. Sadushi-Kolici R, Skoro-Sajer N, Zimmer D et al. Long-term treatment, tolerability, and survival with sub-cutaneous treprostinil for severe pulmonary hypertension. J Heart Lung Transplant 2012; 31 (7): 735-743.

Received December 3, 2019 Accepted February 18, 2020 